Relations industrielles

Industrial Relations

\title{
Absence de dépôt de la convention - Inexistence delà convention vis-à-vis des tiers
}

Volume 17, numéro 2, avril 1962

URI : https://id.erudit.org/iderudit/1021637ar

DOI : https://doi.org/10.7202/1021637ar

Aller au sommaire du numéro

Éditeur(s)

Département des relations industrielles de l’Université Laval

ISSN

0034-379X (imprimé)

1703-8138 (numérique)

Découvrir la revue

Citer ce document

(1962). Absence de dépôt de la convention - Inexistence delà convention vis-à-vis des tiers. Relations industrielles / Industrial Relations, 17(2), 181-184. https://doi.org/10.7202/1021637ar
Résumé de l'article

Le défaut de déposer une convention collective de travail selon les prescriptions de l'article 19 de la Loi des Relations ouvrières de Québec, donne ouverture à la présentation d'une requête en reconnaissance de la part d'une association non signataire en vue d'être reconnue aux lieu et place d'une autre association qui était signataire de la dite convention.
Tous droits réservés @ Département des relations industrielles de l’Université Laval, 1962
Ce document est protégé par la loi sur le droit d'auteur. L'utilisation des services d'Érudit (y compris la reproduction) est assujettie à sa politique d'utilisation que vous pouvez consulter en ligne.

https://apropos.erudit.org/fr/usagers/politique-dutilisation/ 


\section{JURISPRUDENCE DU TRAVAIL}

\section{ABSENCE DE DEPOT DE LA CONVENTION - INEXISTENCE DE LA CONVENTION VIS-A-VIS DES TIERS -}

Le défaut de déposer une convention collective de travail selon les prescriptions de l'article 19 de la Loi des Relations ouvrières de Québec, donne ouverture d̀ la présentation d'une requête en reconnaissance de la part d'une association non signataire en vue d'être reconnue aux lieu et place d'une autre association qui était signataire de la dite convention. 1

(1) Le Syndicat national des travailleurs de l'industrie du camionnage, requérant, et l'Union des camionneurs, hommes d'entrepôt et métiers alliés, local 121, intimée, et Simard Express mis en cause: M. le juge Théodore Lespérance, président, Me K.G.K. Baker, c.r., Me Claude Lavery, MM. Léo M. Côté et André Roy, commissaires; Commissions des Relations ouvrières de Québec, D-175, 13 octobre 1961. Me Marcel Simard, pour le requérant; Me Paul-Etienne Bernier, pour l'intimée; Me Emilien Desbiens, pour la mise-en-cause.

\section{LES FATTS}

Le 27 mars 1961, le requérant présenta une requête en reconnaissance en vue d'être reconnu comme représentant collectif « de tous les employés de Simard Express des Terminus de Chicoutimi et de Québec» (staff intérieur) du bureau d'administration. Cette requête fut déposée aux bureaux de la Commission à Québec le même jour.

Au soutien de sa requête, le requérant alléguait qu'aucune convention collective n'ayant été déposée par l'intimée en temps approprié conformément à l'article 19 de la Loi, il lui était loisible de présenter une requête en vue d'être reconnu aux lieu et place de cette dernière.

Le 14 avril 1961, l'intimée contesta la requête du requérant en alléguant que, celle-ci étant dûment reconnue par la Commission de relations ouvrières pour représenter «tous les chauffeurs et aides-chauffeurs à l'exception des personnes automatiquement exclues par l'article 21 paragraphe « a sous-paragraphe 1o, 2o, 3o, de la Loi » à l'emploi du mis-en-cause, elle avait conclu le 24 spetembre 1960 une convention collective de travail avec ledit mis-en-cause, convention collective dont la durée s'étendait du ler janvier au 31 décembre 1961. Elle alléguait que «aucune requête en reconnaissance syndicale ne peut être accueillie par la Commission, si ce n'est du soixantième au trentième jour précédant l'expiration de ladite convention collective de travail tel qu'il ressort des dispositions de l'article 16 de la Loi. L'intimée concluait sa contestation en demandant qu'audition lui soit accordée pour faire valoir ses moyens et demandait le rejet de la requête du requérant. 
La mise-en-cause ne fait aucune représentation quant à la requête du requérant.

\section{LE DROTT}

... L'intimée a plaidé que la requête soumise par le requérant devait être rejetée, parce qu'elle n'avait pas été présentée dans les délais prévus à l'article 16 de la Loi.

Le procureur du requérant a plaidé que, selon l'article 19 de la loi l'intimée se devait de faire le dépót de la convention collective sans délai, qu'il a négligé de le faire et que, au moment de la présentation de la requête, le 27 mars 1961, soit environ six mois après sa signature, ce dépot n'était pas fait et que, en conséquence, la requête en reconnaissance du requérant est bien fondée. Il ajoutait que la Commission devant juger à la date de la présentation de la requête, elle ne peut considérer une convention qu'elle n'avait pas devant elle à ce moment et qui, au surplus, à cette date n'avait aucun effet. Tout le litige dans la présente affaire tourne autour du dépôt de la convention collective de travail. Les faits qui s'y rapportent sont simples et non contestés. La requête a été déposée à la Commission le 27 mars 1961 et le dépôt de la convention intervenue entre l'intimée et la mise-en-cause le 24 septembre 1960, fut fait le 28 ou 29 mars 1961.

La question à trancher est la suivante: Est-ce que le défaut de déposer une convention collective de travail selon les prescriptions de l'article 19 de la Loi donne ouverture à la présentation d'une requête en reconnaissance de la part d'une association non signataire en vue d'être reconnue aux lieu et place d'une autre association qui était signataire de ladite convention?

De l'analyse de la Loi, la commission en vient à la conclusion que tous ces articles forment un ensemble et qu'ils se tiennent en quelque sorte, que le défaut de suivre les prescriptions de l'article 19 ou de l'article 19a peut avoir pour effet de faire disparaître les restrictions stipulées à l'article 16. L'article 19 ne nous paraît pas avoir d'autre raison d'être en regard de la Loi que de permettre à la Commission d'être saisie de l'existence d'une convention collective pour être en mesure d'appliquer l'article 16 et de permettre aux tiers de se renseigner sur l'existence et la durée de telle convention. C'est pourquoi l'article est impératif et que la responsabilité ou le fardeau du dépôt est imputé à l'association signataire. C'est à elle qu'il incombe de protéger vis-à-vis des tiers sa convention collective en procédant «sans délai » à son dépôt.

Reste l'expression «sans délai » qu'il faut interpréter. «Sans délai » signifie le plus rapidement possible. Sans doute, permet-elle une certaine latitude, mais, dans les circonstances, il appartenait à l'association intimée de prouver qu'elle avait été empêchée de faire ce dépôt avant la date du 28 mars 1961, ce qui n'a pas été fait dans la présente cause.

De plus, le dépôt de la conventoin a été fait dans la présente cause après le dépôt de la requête du requérant, de telle sorte que, au moment où la Commission est légalement appelée à se prononcer les prescriptions de l'article 19 n'ont pas été 
remplies et que la convention est inexistante pour la Commission et pour les tiers.

Ceci ressort du deuxième alinéa de l'article 19, stipule que la « convention collective ne prend effet qu'à compter de ce dépôt ».

Dans les circonstances, la Commission ne peut en arriver qu'à la conclusion que, à la date de la requête, il n'existait pas vis-à-vis des tiers de convention collective de travail et qu'elle doit considérer la requête au mérite, c'est-à-dire s'assures du caractère représentatif de l'association requérante et de son droit à être reconnue.

Sur ce point, la Commission, après étude de ses dossiers a constaté que le caractère représentatif de l'association requérante est acquis de même que son droit à être reconnue.

D’autre part, l'enquête a révélé que le 27 mars 1961, date de la présentation de la requête, le requérant avait fait parvenir à l'intimée 19 démissions de membres de l'intimée ce qui avait pour effet de lui faire perdre son caractère représentatif.

\section{LA DECISION}

En conséquence:

La Commission DECIDE:

10:- d'ANNULER à toutes fins que de droit le certificat de reconnaissance émis en faveur de l'intimée le 25 novembre 1959 pour représenter \& tous les chauffeurs ou aides-chauffeurs à l'exception des personnes automatiquement exclues par l'article 2 et 3 de la Loi ».

2o:- de RECONNAITRE le Syndicat national des travailleurs de l'industrie du camionnage comme représentant collectif du groupe de salariés suivants:

«les salariés au sens de la Loi, à l'exception des employés de bureau ».

A L'EMPLOI DE: Simard Express, Limitée, à ses terminus de Québec et de Chicoutimi.

NOTE OF Me K. G. K. BAKER

Article 19 of the Act imposes an obligation upon a certified association which has entered into a collective labour agreement to transmit forthwith copies thereof to the Board for deposit. The agreement shall not take effect until such deposit is made.

It is obviously in the interests of any such association to fulfill this obligation. The other party to the agreement, the employer, after negotiating in good faith has likewise its own interests to protect. It is my interpretation of the law that while there is no obligation on the part of the employer to make the deposit, he has the right to do so if he wishes. But the law does not so provide in explicit terms. 
The employer, mise-en-cause, made no representations in the present case although the opportunity of doing so was available to him. However, I do consider that the provisions of the law are somewhat anomalous in so far as concerns the deposit of collective labour agreements; and that an amendment should be made to provide explicitly that any party to a collective labour agreement shall have the right to make such deposit.

QUEBEC, October 13th, 1961.

NOTES DE Me CLAUDE LAVERY: Je partage l'opinion de mon collègue, Me Baker.

\section{NÉGOCIATIONS - REFUS DE NÉGOCIER DE BONNE FOI - INTERPRETATION DES ARTICLES 11 ET 42 DE LA LOI DES RELATIONS OUVRIËRES DE QUÉBEC}

- La Cour du Banc de la Reine décide qu'en labsence de preuve établissant hors de tout doute raisonnable, le refus d'une partie de négocier de bonne foi une convention collective de travail, l'article 42, de la loi des Relations ouvrières de Québec ne peut s'appliquer. En matière pénale et criminelle, il ne suffit pas qu'il soit possible ou probable qu'un accusé ait commis une infraction, mais il faut que la Cour en soit moralement convaincue. ${ }^{1}$

Le 22 juillet 1960, le Syndicat des employés de l'alimentation et du commerce de St-Anselme (le présent appelant) a porté plainte contre la Coopérative Dorchester (l'intimé) l'accusant, étant employeur et ayant reçu l'avis prescrit, d'avoir fait défaut, entre le 17 février 1960 et le 22 juillet de la même année, de négocier de bonne foi avec les représentants d'une association de salariés à son emploi reconnue à cette fin par la Commission des relations ouvrières, savoir le plaignant, une convention collective de travail contrairement aux dispositions de l'art. 42 de la Loi des Relations ouvrières, S. R. Q. 1941, chap. 162a (8. Geo. VI. 1944, chap. 30) et amendements.

Après une longue enquête, M. le Juge Albert Dumontier a renvoyé la plainte et acquitté la corporation-intimée.

Le Syndicat appelle de cette décision.

\section{LES FATTS}

Le 28 janvier 1960, le syndicat-appelant avisait l'intimée que ses représentants la rencontrerait pour négocier une convention collective de travail le 9 février

(1) Le Syndicat des Employés de l'alimentation et du Commerce de St-Anselme, Plaignant-appelant vs La Coopérative Dorchester, Accusée-intimée; Cour du Banc de la Reine. Présent: Hon. Léon Casgrain, J.C.S.; No C.B.R. 28-130; Germain, Pigeon, Thibaudeau et Lesage, pour l'appelant; Denys Aubé, pour l'intimée. 